\title{
Editorial
}

\section{Mobility for the Internet of Things}

\author{
Bartolomeo Montrucchio $\left(\mathbb{D},{ }^{1}\right.$ Sorin-Aurel Moraru $\mathbb{D D}^{2}$ Claudiu Pozna $\mathbb{D D}^{2,3}$ \\ and Javier Vales Alonso $\left.{ }^{4}\right)^{4}$ \\ ${ }^{1}$ Dipartimento di Automatica e Informatica, Politecnico di Torino, Corso Duca degli Abruzzi 24, 10129 Torino, Italy \\ ${ }^{2}$ Department of Automatics and Information Technology, Transilvania University of Brasov, Str. Mihai Viteazu Nr. 5, \\ 500174 Brasov, Romania \\ ${ }^{3}$ Department of Informatics, Széchenyi István University, Egyetem tér 1, 9026 Györ, Hungary \\ ${ }^{4}$ Department of Information Technologies and Communications, School of Telecommunication Engineering, \\ Technical University of Cartagena, Plaza del Hospital 1, 30202 Cartagena, Spain
}

Correspondence should be addressed to Bartolomeo Montrucchio; bartolomeo.montrucchio@polito.it

Received 18 April 2018; Accepted 18 April 2018; Published 14 May 2018

Copyright (C) 2018 Bartolomeo Montrucchio et al. This is an open access article distributed under the Creative Commons Attribution License, which permits unrestricted use, distribution, and reproduction in any medium, provided the original work is properly cited.

Internet of Things (IoT) currently delivers data communication services to a broad set of devices in many realms, such as Industry 4.0, self-driven cars, and sensor networks. Many of those devices intrinsically work over mobile systems or evolve toward mobility or both because they move with humans as is the case of smartphones or because they move by themselves; in the latter case, it can be possible to see robots for home managing or selfdriven cars or UAVs, maybe to deliver packets at home. Following the Call for Papers, this special issue has focused on managing mobility in IoT, both from a theoretical and a practical point of view.

In the paper "An Energy-Aware Task Offloading Mechanism in Multiuser Mobile-Edge Cloud Computing," the problem of the offloading decision is formulated as a $0-1$ nonlinear integer program under the constraints of the channel interference threshold and the time deadline. Through the classification and priority determination for the mobile devices, a reverse auction-based offloading method is proposed to solve this optimization problem for energy efficiency improvement. In fact, the mobile-edge cloud computing, an emerging and prospective computing paradigm, can facilitate the complex application execution on resource-constrained mobile devices by offloading computation-intensive tasks to the mobile-edge cloud server, which is usually deployed in close proximity to the wireless access point. However, in the multichannel wireless interference environment, the competition of mobile users for communication resources is not conducive to the energy efficiency of task offloading.
In the paper "A New Theoretical Approach: A Model Construct for Fault Troubleshooting in Cloud Computing," the authors propose a new theoretical algorithm to construct a model for fault troubleshooting. In cloud computing, there are four effective measurement criteria: (i) priority, (ii) fault probability, (iii) risk, and (iv) the duration of the repair action determining the efficacy of troubleshooting. The authors combine a naïve Bayes classifier (NBC) with a multivalued decision diagram (MDD) and an influence diagram (ID), which structures and manages problems related to unambiguous modeling for any connection between significant entities. First, the NBC establishes the fault probability based on a naïve Bayes probabilistic model for fault diagnosis. This approach consists of three steps: (i) identifying the network parameters to show the reliance for probability relationship among the entire set of nodes; (ii) determining the structure of the network topology; and (iii) assessing the probability of the fault being propagated. This calculates the probability of each node being faulty given the evidence. Second, the MDD combines the influential factors of four measurements and determines the utility value of prioritizing their actions during each step of the fault troubleshooting which in turn assesses which fault is selected for repair. The authors demonstrate how the procedure is adapted by their method, with the host server's failure to initiate a case study. This approach is highly efficient and enables low-risk fault troubleshooting in the field of cloud computing. 
In the paper "Multihoming for Mobile Internet of Multimedia Things," mobile IoT, for example, the "things" that are part of vehicular or public transportation systems, is used as a scenario for showing the possibility of using the LISP (Locator Identifier Separation Protocol) for multihoming and load-balancing purposes. The authors have implemented and tested a demonstrator of a mobile LISP IoT gateway that is also integrated with cloud-based video analytics. Mobility, redundancy, and bandwidth requirements are transforming the communication models used for IoT, mainly in case of critical communications and multimedia streaming ("IoMT, Internet of Multimedia Things"), as wireless video traffic is expected to be $60-75 \%$ of the global mobile traffic by 2020 . One of the characteristics of $5 \mathrm{G}$ networks will be the proliferation of different/ heterogeneous radio networks (virtualized radio access networks, RAN, new energy-efficient radios, femtocells, and offloading capabilities) and the possibility for IoT objects to connect and load balance between dual and multiple RANs.

In the paper "Determination of Collection Points for Disjoint Wireless Sensor Networks," the problem of recovering from a broken wireless sensor network is considered. In fact, when the connection in WSNs is broken, a subset of nodes which serve as the data collection points (CPs) can buffer the data from sensors and transfer these data to mobile data collectors (MDCs) to restore the connectivity of WSNs. One of the existing problems is how to decide the numbers and positions of CPs for obtaining an optimal path of MDC. In order to deal with this problem, a selection method of CPs is proposed to reduce the traveling distance of MDCs. Meanwhile, with this selection method, the changing rules and the stability of the path of MDC are theoretically proved. A 100-node WSN is implemented to test the proposed method. The evaluation results verify that the proposed method is efficient and valuable.

In the paper "Discovering Social Community Structures Based on Human Mobility Traces," the authors consider a community detection problem in a social network. A social network is composed of smaller communities; that is, a society can be partitioned into different social groups in which the members of the same group maintain stronger and denser social connections than individuals from different groups. In other words, people in the same community have substantially interdependent social characteristics, indicating that the community structure may facilitate understanding human interactions and the individual's behaviors. The authors detect the social groups within a network of mobile users by analyzing the Bluetooth-based encounter history from a real-life mobility dataset. Community detection methodology focuses on designing similarity measurements that can reflect the degree of social connections between users by considering tempo-spatial aspects of human interactions, followed by clustering algorithms. The authors also present two evaluation methods for the proposed schemes. The first method relies on the natural properties of friendship, where the longevity, frequency, and regularity characteristics of human encounters are considered. The second is a movement prediction-based method which is used to verify the social ties between users. The evaluation results show that the proposed schemes can achieve high performance in detecting the social community structure.

\author{
Bartolomeo Montrucchio \\ Sorin-Aurel Moraru \\ Claudiu Pozna \\ Javier Vales Alonso
}

\section{Acknowledgments}

Guest editors would like to thank all the authors for having submitted their papers and anonymous reviewers for their time and help. 


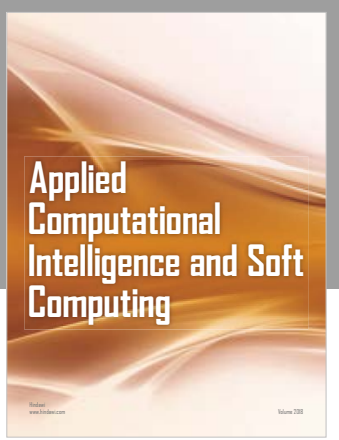

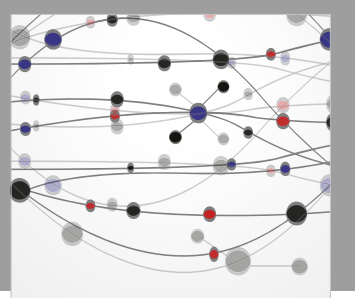

The Scientific World Journal
Submit your manuscripts at

Computing
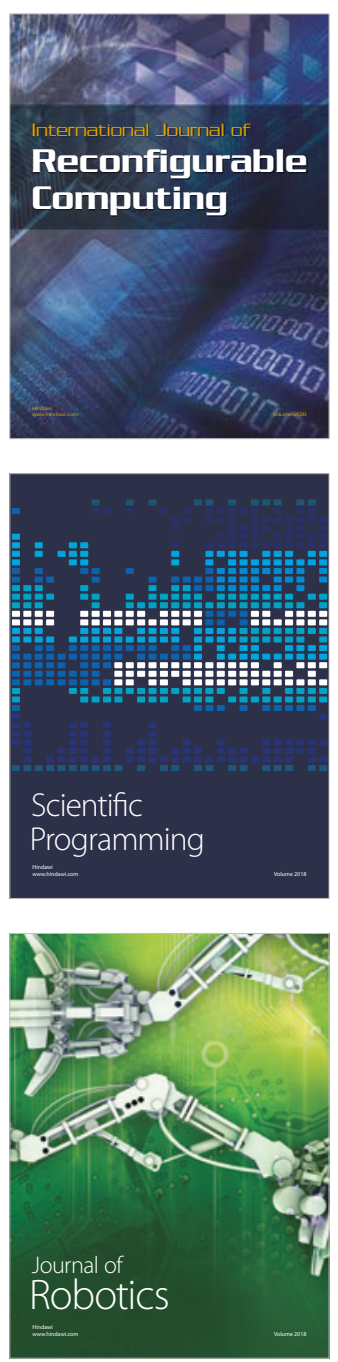

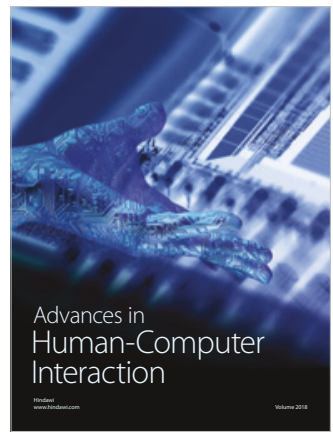

Human-Compute

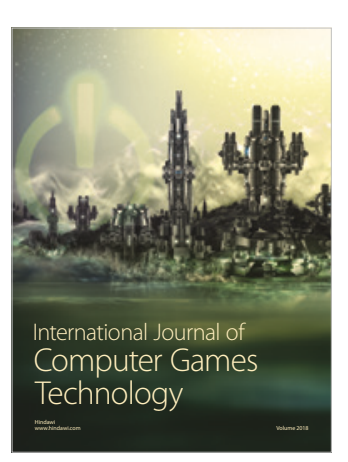

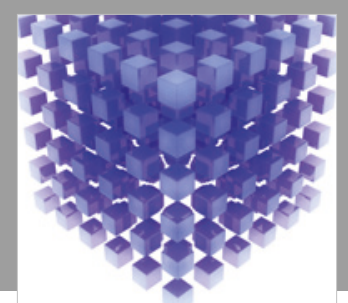

Mathematical Problems in Engineering

\section{Engincering}
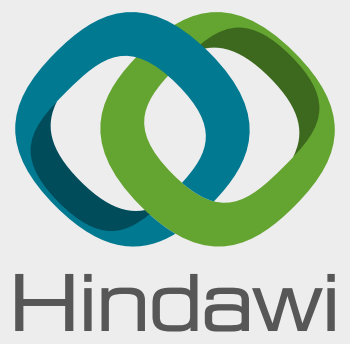

www.hindawi.com
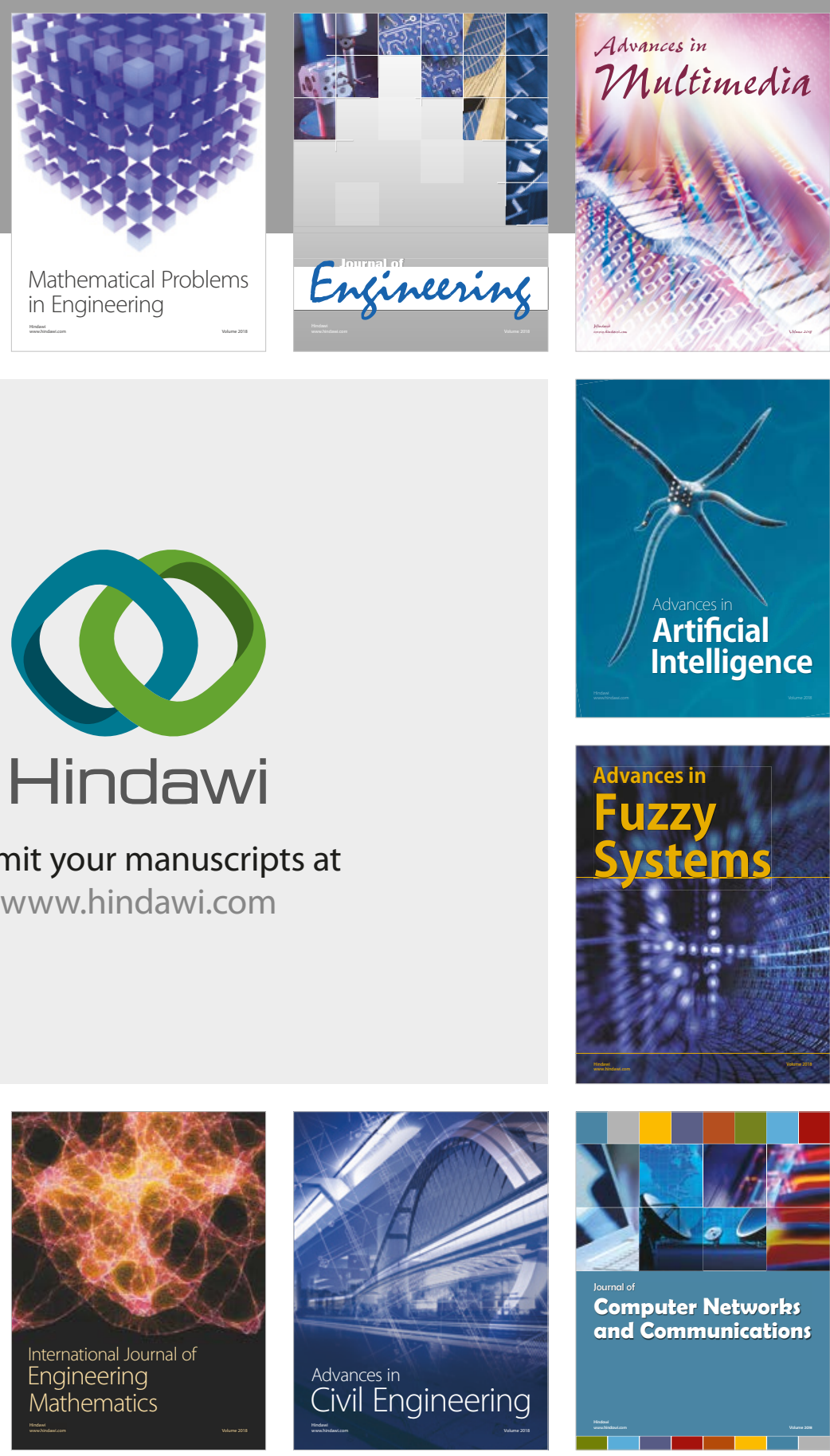

Computer Networks and Communications

Multimedia
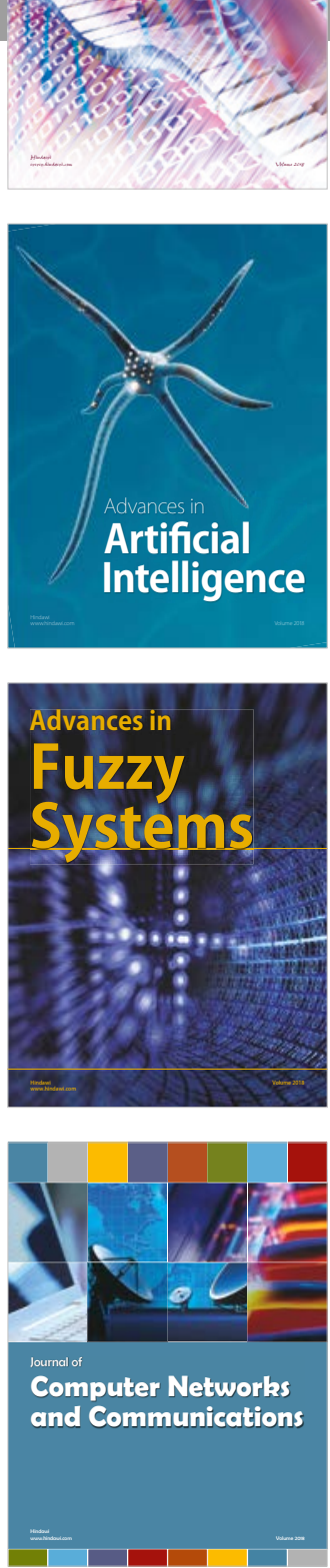

Advances in

Modelling \&

Simulation

in Engineering

interaction

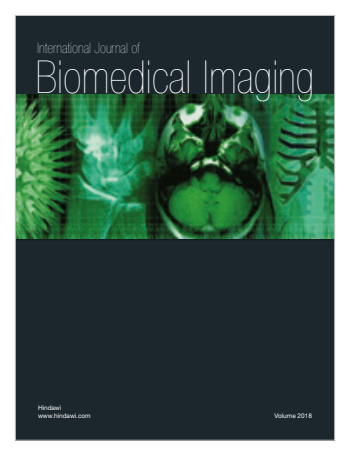

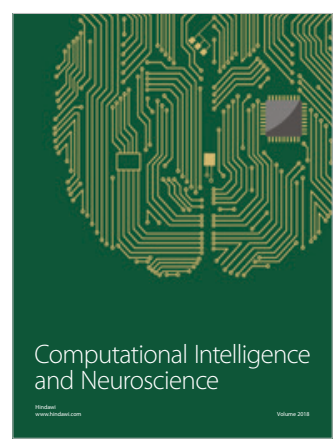

